Konno procedure. ${ }^{5}$ An additional annular enlargement anteriorly also keeps the Manouguian incision to the minimum if needed and minimizes any potential mitral valve dysfunction.

This proposed modified extended 2-patch technique enlarges the proximal aorta in 2 directions from the annulus to ascending aorta. In the context of reoperative aortic reconstruction, it can be used as an alternative to total aortic root replacement and coronary reimplantation.

We thank Ms Angela Butler for the illustrations in this article.

\section{References}

1. Vitale N, Hornung T, Ciotti G, Hamilton JR, Pozzi M, Hasan A. The Ross procedure in children under ten years of age. J Heart Valve Dis. 1999;8:601-4

2. McBrien A, Chaudhari M, Crossland DS, Aspey H, Heads-Baister A, Griselli M et al. Single-centre experience of 101 paediatric and adult Ross procedures: mid-term results. Interact Cardiovasc Thorac Surg. 2012;14:570-4

3. Manouguian S, Seybold-Epting W. Patch enlargement of the aortic valve ring by extending the aortic incision into the anterior mitral leaflet. New operative technique. J Thorac Cardiovasc Surg. 1979;78:402-12.

4. Nicks R, Cartmill T, Bernstein L. Hypoplasia of the aortic root. The problem of aortic valve replacement. Thorax. 1970;25:339-46.

5. Yamaguchi M, Ohashi H, Imai M, Oshima Y, Hosokawa Y. Bilateral enlargement of the aortic valve ring for valve replacement in children. New operative technique. J Thorac Cardiovasc Surg. 1991;102:202-6.

\title{
Anomalous aortic origin of the coronary artery: Does pulmonary artery translocation affect coronary artery course?
}

\author{
Vitor C. Guerra, MD, ${ }^{\mathrm{a}, \mathrm{b}}$ Michael R. Recto, $\mathrm{MD},{ }^{\mathrm{a}, \mathrm{b}}$ Corey Goldman, $\mathrm{MD}, \mathrm{PhD},{ }^{\mathrm{c}}$ and \\ Thomas Yeh, Jr, MD, PhD, ${ }^{\text {a,d }}$ New Orleans, La
}

The debate around timing and choice of procedure for anomalous aortic origin of the coronary artery (AAOCA) is now the subject of an ongoing study by the Congenital Heart Surgeons Society. ${ }^{1}$ Approaches have included unroofing, reimplantation, and pulmonary artery (PA) translocation. ${ }^{2}$ Whether moving the distal PA exerts any effect on the proximal PA, and thereby acts to relieve coronary compression, has been questioned. ${ }^{2}$ Here we analyze preoperative and postoperative imaging in a single patient.

An echocardiogram performed for a murmur in a 12-year-old male football player revealed AAOCA. The patient had neither chest pain nor shortness of breath. Examination and chest radiography were unrevealing. Electrocardiography revealed normal sinus rhythm with early repolarization and increased left-sided forces. Transthoracic echocardiography (and later catheterization) revealed a 3-mm left main coronary artery (LM) arising from the right coronary sinus (Figure 1, $A l$ and $D 1$ ) that shared a common ostium with a 2-mm right coronary artery (Figure 1, Cl). The LM passed between the aorta and PA and gave rise to the left anterior descending and left

\footnotetext{
From the Tulane Pediatric Heart Center, ${ }^{a}$ the Department of Pediatrics, ${ }^{b}$ the Department of Internal Medicine, ${ }^{\mathrm{c}}$ and the Department of Surgery, ${ }^{\mathrm{d}}$ Tulane University School of Medicine, New Orleans, La.

Disclosures: Authors have nothing to disclose with regard to commercial support. Received for publication July 26, 2013; accepted for publication Aug 1, 2013; available ahead of print Sept 30, 2013.

Address for reprints: Thomas Yeh, Jr, MD, PhD, 1430 Tulane Ave, SL-22, New Orleans, LA 70112.

J Thorac Cardiovasc Surg 2013;146:1549-51

$0022-5223 / \$ 36.00$

Copyright (c) 2013 by The American Association for Thoracic Surgery

http://dx.doi.org/10.1016/j.jtcvs.2013.08.010
}

circumflex coronary arteries. There was trivial tricuspid insufficiency, no outflow tract obstruction, and normal chamber dimensions (left ventricular posterior wall, 9 $\mathrm{mm}$; septum, $11 \mathrm{~mm}$ ). Left ventricular function was hyperdynamic (shortening fraction $56 \%$, ejection fraction $86 \%$ ). Computed tomographic (CT) angiography (Figure 1, $E 1$ and $F 1$ ) confirmed the diagnosis and the absence of intramural coronaries. Exercise stress echocardiography (Bruce protocol) was terminated at 10 minutes, 22 seconds for leg fatigue at 12.3 metabolic equivalents. Pulse and blood pressure increased from 83 to196 beats $/ \mathrm{min}$ (94\% of age predicted maximum) and 139/45 to 204/ $41 \mathrm{~mm} \mathrm{Hg}$, respectively. There were no electrocardiographic changes, arrhythmias, or wall motion abnormalities. Threedimensional transesophageal echocardiography revealed neither coronary ostial stenosis nor intramural course (Figure 1, B1).

At surgery, little distance separated the aorta from the PA when dissected down to the coronaries to confirm their anatomy. The branch PAs were dissected to their lobar branches to relieve tension. Under bicaval cannulation with aortic crossclamping, the right PA was transected near its origin and its posterior wall reanastomosed anterior to the ascending aorta. The right PA was generously patched anteriorly with autologous pericardium to avoid narrowing. The patient was weaned easily from cardiopulmonary bypass with good ventricular function. Only when the PA was pressurized did we note an anterior bowing in the PA away from the aorta, undoubtedly facilitated by adventitial lysis. This accentuated arching created an obvious clear space away from the coronaries. 

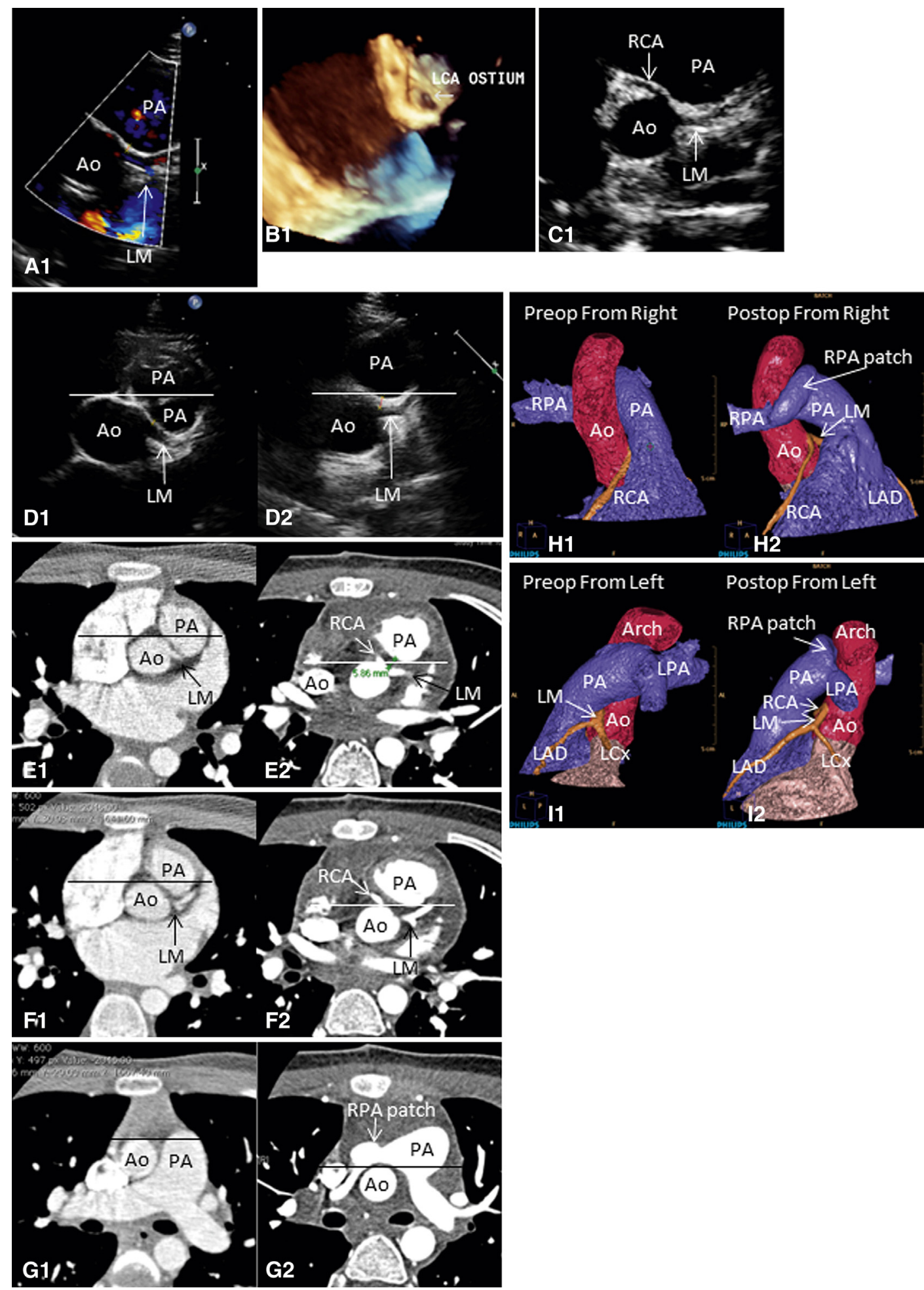

FIGURE 1. A1-I1, Preoperative images; D2-I2, postoperative images. A1, C1, D1, and D2, Transthoracic echocardiography (with color, A1; without color, C1, D1, and D2). B1, Transesophageal echocardiography of left main orifice. E1, E2, F1, F2, G1, and G2, Computed tomographic scans at level of origin of LM (E1 and E2), bifurcation of LM (F1 and F2), and bifurcation of PA (G1 and G2). H1, H2, I1, and I2, Three-dimensional computed tomographic reconstructions viewed from patient's right $(\mathrm{H} 1$ and $\mathrm{H} 2)$ and left (I1 and I2). The resolution differences reflect the use of a 16-slice computed tomographic scan preoperatively and a 64-slice scan postoperatively. PA, Pulmonary artery; $A o$, aorta; $L M$, left main coronary artery; $L C A$, left coronary artery; $R C A$, right coronary artery; $R P A$, right pulmonary artery, $L A D$, left anterior descending coronary artery; $L C x$, left circumflex coronary artery; $L P A$, left pulmonary artery. See text for more complete descriptions. 
In the preoperative echocardiogram (Figure 1,Dl), a line drawn on the anterior border of the ascending aorta bisects the PA. Postoperatively (Figure 1,D2), the body of the PA has clearly moved anterior to that line, away from the $\mathrm{LM}$, whose original posterior curvature is straightened on the postoperative study. CT angiographic sections at the origin of the LM from the aorta (Figure 1, E1 [preoperative] and $E 2$ [postoperative]) and at the level of the bifurcation of the LM into the left anterior descending and left circumflex coronary arteries (Figure 1, F1 [preoperative] and F2 [postoperative]) show the same phenomenon. An increase in distance between the PA and the virtual common origin of the coronaries (preoperative $1 \mathrm{~mm}$, postoperative $5.8 \mathrm{~mm}$ ) is demonstrated (Figure 1, E2). Distally, at the bifurcation of the PA (Figure 1, G1 [preoperative] and G2 [postoperative]), most of the PA is anterior to the aorta. Comparison of preoperative CT reconstructions viewed from the patient's right and left sides (Figure $1, H I$ and $I I$, respectively) with postoperative views (Figure $1, H 2$ and $I 2$, respectively) confirms the anterior bowing of the PA that we noted at surgery, serving to increase the distance between the aorta and
PA. Through that enlarged neoaortopulmonary window, the uncompressed takeoffs of the RCA and LM are seen from both sides (Figure 1, H2 and I2). Before bifurcating, the LM (Figure 1, E2 vs E1 and $F 2$ vs $F 1$ ) takes a straight course postoperatively and has lost the posterior curvature seen preoperatively. We believe that the fulcrum of motion is not the pulmonary annulus, but rather the attachment of the infundibulum to the right ventricular mass. When the PA is foreshortened by moving the bifurcation anteriorly (and closer to that fulcrum), the main PA arches, allowing the proximal main pulmonary artery to move away from the coronaries. To our knowledge, this is the first imaging analysis to demonstrate desirable anatomic effects of PA translocation.

\section{References}

1. Brothers J, Gaynor JW, Paridon S, Lorber R, Jacobs M. Anomalous aortic origin of a coronary artery with an interarterial course: understanding current management strategies in children and young adults. Pediatr Cardiol. 2009; 30:911-21

2. Mainwaring RD, Reddy VM, Reinhartz O, Petrossian E, MacDonald M, Nasirov T, et al. Anomalous aortic origin of a coronary artery: medium-term results after surgical repair in 50 patients. Ann Thorac Surg. 2011;92:691-7.

\title{
Perventricular device closure of ventricular defects in 235 young children: A single-center experience
}

\author{
Hua-Shan Xu, MD, ${ }^{a}$ Kamran Yunus Inamdar, MM, ${ }^{\mathrm{b}}$ Khan Mohammed Firoj, MM, ${ }^{\mathrm{a}}$ and \\ Wen-Zeng Zhao, MM, ${ }^{\mathrm{a}}$ Zhengzhou, Henan, and Urumqi, Xin Jiang, China
}

Perventricular device closure of ventricular septal defect (PDCVSD) is no longer a novel modality, ${ }^{1,2}$ and current research is focused on the indications and long-term outcomes of the technique. ${ }^{3}$ We report 235 ventricular septal defect (VSD) cases with indications for PDCVSD from September 2010 to May 2013.

\section{MATERIALS AND METHODS}

PDCVSD was indicated for perimembranous, subarterial, or muscular VSD when the defects were 4 to $8 \mathrm{~mm}$ in size and $2 \mathrm{~mm}$ away from the arterial valve. Of 235 cases, 203 were perimembranous and 32 were

\footnotetext{
From the Cardiovascular Surgery Department, ${ }^{a}$ The First Affiliated Hospital of Zhengzhou University, Institute of Clinical Medical Research of Universities Henan, Zhengzhou, Henan, China; and Cardiovascular Surgery Department, ${ }^{\text {b }}$ The First Affiliated Hospital of Xinjiang Medical University, Urumqi, Xin Jiang, China.

Disclosures: Authors have nothing to disclose with regard to commercial support.

Received for publication June 18, 2013; revisions received July 31, 2013; accepted for publication Aug 9, 2013; available ahead of print Sept 26, 2013.

Address for reprints: Hua-Shan Xu, MD, Cardiovascular Surgery Department, The First Affiliated Hospital of Zhengzhou University, Zhengzhou 450052, Henan,

China (E-mail: xhsay@hotmail.com).

J Thorac Cardiovasc Surg 2013;146:1551-3

0022-5223/\$36.00

Copyright (c) 2013 by The American Association for Thoracic Surgery

http://dx.doi.org/10.1016/j.jtcvs.2013.08.004
}

subarterial; patient ages ranged from 6 months to 12 years and weights from 6 to $36 \mathrm{~kg}$. Occluder size (waist) was 2- to $4-\mathrm{mm}$ larger than the defect size, as measured using transesophageal echocardiogram. A symmetric occluder was the default choice, but an asymmetric occluder was selected when a defect was near the aortic valve. ${ }^{4}$

\section{SURGICAL PROCEDURE}

A transesophageal echocardiogram probe is placed in a supine patient after anesthesia administration. A lower midline incision $(3-4 \mathrm{~cm})$ is made and the lower sternum is sawed open. The pericardium is cut and suspended. A 4-0 prolene purse-string suture is placed in the selected puncture spot. Heparin is administered $(1 \mathrm{mg} / \mathrm{kg})$ via a central venous pressure line. A guidewire is introduced into the right ventricle and through the defect into the left ventricle, and a catheter is then introduced via the guidewire into the left ventricle to form a passage. The occluder is inserted into the defect and opened. After confirming that residual shunt and tricuspid and aortic valve regurgitations are absent, the occluder is released. The purse-string suture is knotted, and the chest is closed using a pericardial drainage tube. Protamine is not used. The surgery lasts 20 to 60 minutes. Aspirin is 